This paper was presented at a colloquium entitled "Quasars and Active Galactic Nuclei: High Resolution Radio Imaging," organized by a committee chaired by Marshall Cohen and Kenneth Kellermann, held March 24 and 25, 1995, at the National Academy of Sciences Beckman Center, Irvine, CA.

\title{
Superluminal sources
}

[cosmology (observations)/galaxies (jets)/quasars (general)/radio continuum (galaxies)]

\author{
R. C. Vermeulen \\ Owens Valley Radio Observatory, California Institute of Technology 105-24, Pasadena, CA 91125
}

\begin{abstract}
Predictions for the apparent velocity statistics under simple beaming models are presented and compared to the observations. The potential applications for tests of unification models and for cosmology (source counts, measurements of the Hubble constant $H_{0}$ and the deceleration parameter $q_{0}$ ) are discussed. First results from a large homogeneous survey are presented. The data do not show compelling evidence for the existence of intrinsically different populations of galaxies, BL Lacertae objects, or quasars. Apparent velocities $\beta_{\text {app }}$ in the range 1-5 $h^{-1}$, where $h=$ $H_{0} / 100 \mathrm{~km} \cdot \mathrm{s}^{-1} \cdot \mathrm{Mpc}^{-1}\left[1\right.$ megaparsec $\left.(\mathrm{Mpc})=3.09 \times 10^{22} \mathrm{~m}\right]$, occur with roughly equal frequency; higher values, up to $\beta_{\text {app }}$ $=10 h^{-1}$, are rather more scarce than appeared to be the case from earlier work, which evidently concentrated on sources that are not representative of the general population. The $\beta_{\text {app }}$ distribution suggests that there might be a skewed distribution of Lorentz factors over the sample, with a peak at $\gamma_{\mathbf{b}} \approx$ $2 h^{-1}$ and a tail up to at least $\gamma_{b} \approx 10 h^{-1}$. There appears to be a clearly rising upper envelope to the $\beta_{\text {app }}$ distribution when plotted as a function of observed 5-GHz luminosity; a combination of source counts and the apparent velocity statistics in a larger sample could provide much insight into the properties of radio jet sources.
\end{abstract}

\section{Introduction}

Superluminal motion studies and "High Resolution Radio Imaging of Quasars and Active Galactic Nuclei (AGN)" have been narrowly intertwined almpst from the outset (1-4), even though the modern term superluminal was only coined a decade later (5), and even though originally most people seemed to have only expanding spheres rather than jets in mind (6). While there is no opportunity here for a historical review, it is appropriate to remind everyone of the prominent role played by the organizers of this conference, Marshall Cohen and Ken Kellermann. I am privileged to be able to collaborate with both of them on studies of superluminal motion.

This review will not deal with the wealth of information that has been gathered on a select few sources, such as 3C $345(7-9)$, 4C 39.25 (10), or BL Lacertae (BL Lac) (11). In such famous sources, intensive and prolonged monitoring has led to substantial insight into the jet kinematics, and a large, detailed body of data is available to confront hydrodynamical model calculations of jets and shocks. This review in some sense takes the opposite approach in using data for many more sources but including relatively sparse observations (for many objects the bare minimum of a snapshot image at two epochs). As long as the source selection criteria are well known, this approach has

The publication costs of this article were defrayed in part by page charge payment. This article must therefore be hereby marked "advertisement" in accordance with 18 U.S.C. $\$ 1734$ solely to indicate this fact. the virtue that population models can be constructed involving a distribution of Lorentz factors, jet bending, pattern motions, acceleration, or whatever other complexity is thought to be indicated by the data. These models can be compared to the observed distribution of (superluminal) apparent velocities with the help of Monte Carlo simulations. Thus, as long as the data are obtained and used in a homogeneous fashion, the apparent velocity statistics of the sample reveal for the population as a whole complexities that are missed in individual objects.

In this review (superluminal) velocities or upper limits are only used if derived from an observed change in separation between two recognizable features in a radio source. This excludes values based only on the timing of the appearance of a new knot and/or a radio flare. The inferences drawn from this kinematic approach can then be compared to other indicators of relativistic motion and a small angle to the line of sight, such as variability $(12,13)$ and a high brightness temperature (14), a high ratio of core to extended radio luminosity (15), detectable $\gamma$-rays $(16,17)$, a deficit of inverse-Compton $\mathrm{x}$-rays (18), or the shape of radio source count functions (19). Throughout this review, Friedmann cosmology is used with the Hubble constant $H_{0}=100 h \mathrm{~km} \cdot \mathrm{s}^{-1} \cdot \mathrm{Mpc}^{-1}$ [1 megaparsec $\left.(\mathrm{Mpc})=3.09 \times 10^{22} \mathrm{~m}\right]$. Where appropriate the dependence of the results on $h$ and the deceleration parameter $q_{0}$ is shown. Indeed, since superluminal radio sources can be observed over a wide range of redshifts, their statistics can contribute to a discrimination between different cosmological models (20).

\section{Randomly Oriented Sources}

The model predictions in this review, based mostly on Monte Carlo simulations, follow the formalism and assumptions outlined earlier (21). In particular, the radio jets are assumed to be narrow and (in the first instance) straight, to have random intrinsic orientations, and to have no relationship between the Lorentz factor $\lambda$ and the intrinsic (isotropic) luminosity. Under these circumstances, it follows immediately from the solid angle available that many jets will be pointed near the plane of the sky and show apparent velocities $\beta_{\text {app }}=v_{\text {app }} / c \approx 1$, where $c$ is the speed of light. There will be a modest fraction of superluminals from jets at angles of $\theta \sim 1 / \gamma$ and a small percentage of knots with $\beta_{\text {app }}<1$ in jets pointed almost straight towards us. This distribution of velocities is shown in Fig. 1.

2.1. Cosmological Parameters. In principle, it should be possible to use a sample of randomly oriented relativistic jet

Abbreviations: AGN, active galactic nuclei; BL Lac, BL Lacertae; Jy, Jansky; pc, parsec; FR-I, Fanaroff-Riley type I radio source; VLBI, very-long-baseline radio interferometry; PR, Pearson-Readhead VLBI survey; CJ, California Institute of Technology-Jodrell Bank VLBI survey. 
sources with a range of redshifts-for example, selected on their low-frequency radio emission-to measure both $h$ and $q_{0}$, by using the predominant observed apparent velocity [or perhaps the lower limit (22)] as a standard velocity of $c$ or of $2 c$ if separating features from opposite jets are observed. No appropriate samples have currently been studied well enough. It would be even better if features with a known common origin separating from each other in oppositely directed relativistic jets can be found. By using the arm-length or perhaps brightness ratio in addition to the apparent motions, the jet velocity and angle to the line of sight can be obtained separately. Some of the Compact Symmetric Objects (23) are promising in this regard; in particular, there is a detection of bidirectional motion in $1946+708$ (24).

2.2. AGN Unification. Given values for $h$ and $q_{0}$, motion statistics can also be used to test unification models. For example, a high fraction of superluminals in a sample would indicate jets oriented predominantly at small-to-moderate angles to the line of sight. Several groups are pursuing the apparent velocity statistics of complete samples of quasars selected at low frequency, which minimizes orientation bias $(25,26)$. This is hard work because many of the cores are quite weak [down to 1 milliJansky (mJy), in agreement with the expectation that their emission is Doppler beamed away from us (27)]. Indeed, the current statistics (21) do uphold the trend of decreasing apparent velocities for sources with decreasing core fraction $(R)$. However, there is much scatter (28), and the trend is primarily seen in the upper envelope to the observed motions.

At the very largest measured values of core-dominance, which often occur in BL Lac objects, it seems that the apparent velocities may decrease again, as expected for jets viewed within the $1 / \gamma$ beaming cone. The statistics on high-luminosity BL Lacs therefore do not require the assertion that they are intrinsically different from the core-dominated quasars (29, 30). The possibly characteristic perpendicular polarization structure of BL Lac jets $(29,30)$ may result from preferential viewing of differently polarized regions than in the quasars $(31$, 32). Indeed, both regions of parallel and perpendicular polarization have now been found in 3C345 (9). The lower luminosity BL Lac objects probably should be compared to FR-I (Fanaroff-Riley type I radio source) galaxies (33), but while the apparent velocities in general are slower than in more powerful objects (see also $\$ 4.1, \S 4.4$ ), there are not enough motions known to constrain this brand of unification.

If there were a range of different Lorentz factors within each single jet-for example, a fast core and a subrelativistic cocoon-as has been discussed in the context of FR-I jets (31, 34 ), then $\beta_{\text {app }}$ observed for a given orientation will be that for which Doppler boosting is most favorable: a high value for jets close to the line of sight, lower values for jets pointed further away, and potentially $\beta_{\text {app }}=0$ for jets in the plane of the sky. This has important consequences when exploring population models in which there is a cutoff angle to the line of sight [e.g., $\theta_{\max }=45^{\circ}$ for quasars (35)]. As shown in Fig. 2, the predictions assuming a single jet $\gamma$ and a given angle $\theta_{\max }$ are degenerate with the predictions for $\theta_{\max } / 2$ and assuming there is a wide range of Lorentz factors associated with each jet. If, for example, $\beta_{\mathrm{app}} \approx 1$ is found to be predominant in a sample of lobe-dominated quasars, this does not by itself prove that the sample is randomly oriented without any exclusion angle but could instead be accommodated under a $\theta_{\max }=45^{\circ}$ hypothesis if radiating matter moving at a modest $\beta \approx 1 / \sqrt{2}$ exists in most jets.

\section{Samples Selected on Beamed Emission}

Again as in earlier work (21), the differential source counts are assumed to be a power law function of intrinsic flux density, with index 2.5. It is assumed that in a complete flux-limited sample at high radio frequency, selection of sources from a population of randomly oriented jets takes places through Doppler favoritism. Only flat-spectrum continuous jets are considered, for which Doppler boosting depends on the Doppler factor to the power 2.0; this could be rather different with other assumptions (36).

3.1. Upper $\boldsymbol{\beta}_{\text {app }}$ Envelope. If the Lorentz factor associated with the observed moving radio knots $\left(\gamma_{\mathrm{p}}\right)$ is the same as that of the bulk flow $\left(\gamma_{\mathrm{b}}\right)$, then the width of the beaming $\operatorname{cone}(\sin \theta$ $\left.\sim 1 / \gamma_{\mathrm{b}}\right)$ is well matched to the angle at which the largest apparent motion occurs $\left(\sin \theta=1 / \gamma_{\mathrm{p}}\right)$. Thus, many fast motions are expected in samples selected on beamed emission. Fig. 1 shows the apparent velocity distribution predicted for a sample selected on beamed emission with a single value of $\gamma_{p}$ $=\gamma_{\mathrm{b}}$ for all jets. It is in marked contrast to the distribution expected for randomly oriented jets. Most of the observed

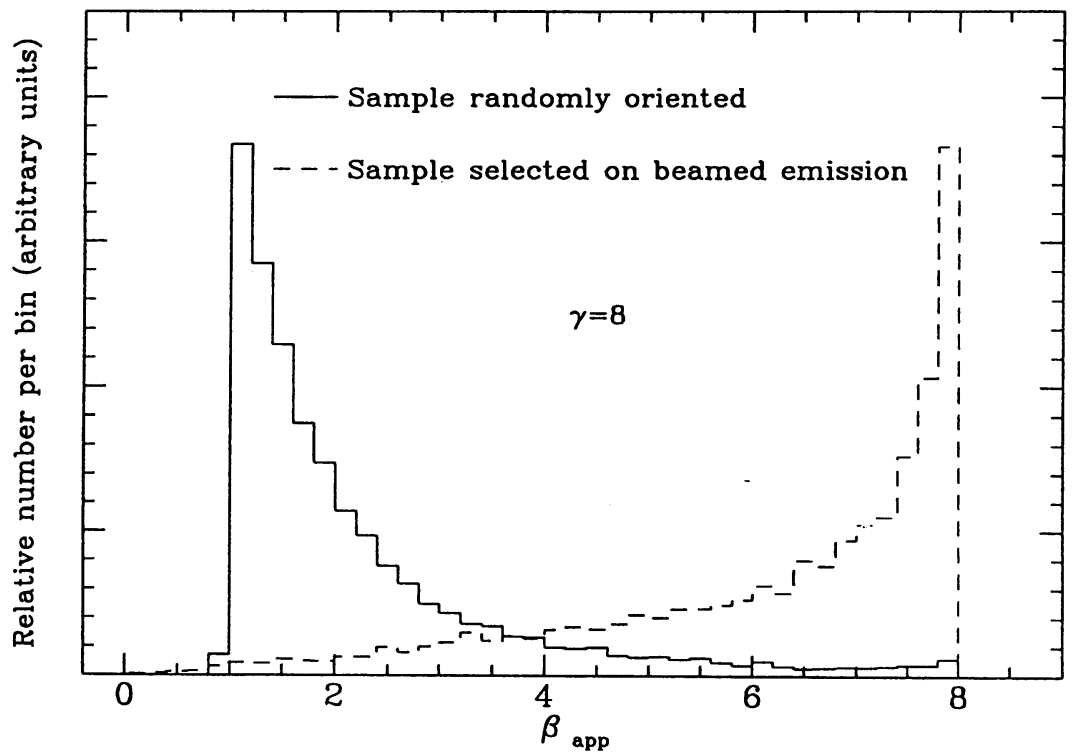

Fig. 1. Predicted apparent velocity distributions for a randomly oriented sample (solid line) and for a sample selected on Doppler-boosted emission (broken line). A single bulk and pattern Lorentz factor is assumed, $\gamma=8$, but the value chosen does not affect the global shape of the predicted distributions. 


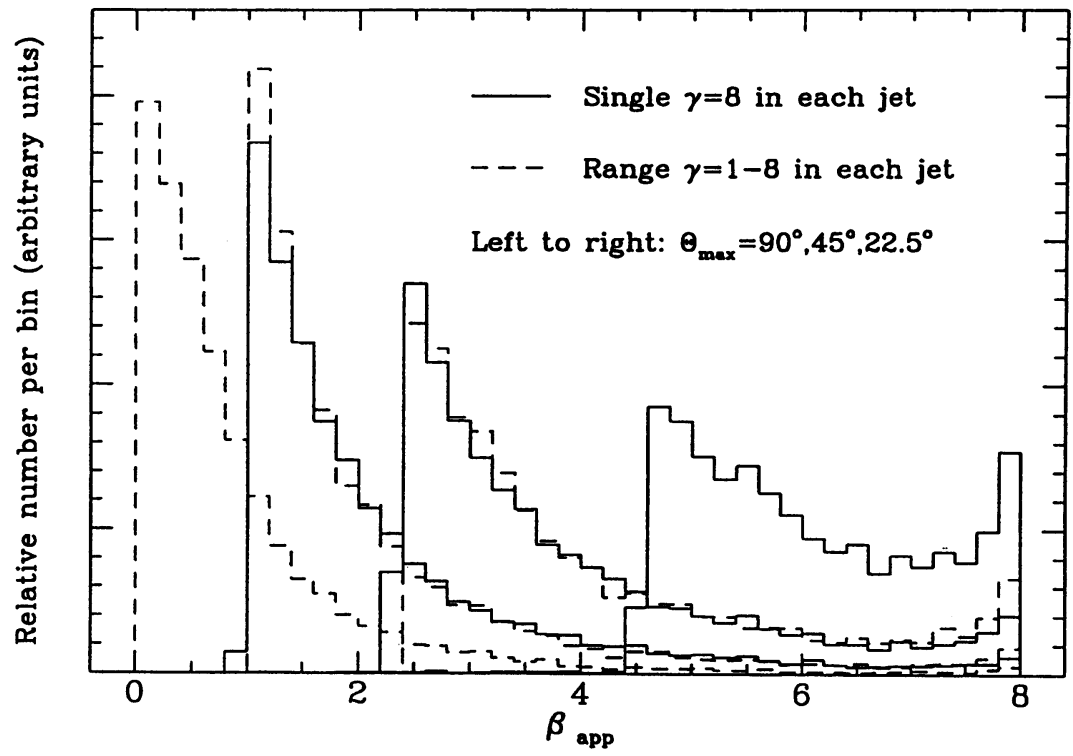

FIG. 2. For randomly oriented samples, assumption of a progressively more restrictive upper cutoff in the distribution of angles to the line of sight $\left(\theta_{\max }\right)$ shifts the predicted apparent velocity distribution to progressively higher values. On the other hand, assumption of a range of Lorentz factors in each jet (broken lines) shifts the predicted apparent velocity distribution to lower values than with a single Lorentz factor (solid lines). There is a degeneracy in the predictions that may hamper tests of unification models. The global shape of the distributions does not depend on the specific choice $\gamma=8$.

motions should now be slightly below the maximum possible velocity $\left(\beta_{\mathrm{app}}=\beta_{\mathrm{p}} \gamma_{\mathrm{p}}\right)$, which should be a sharply defined upper edge. The match between optimal beaming and large motion occurs for all relativistic jets, and when normalized to $\beta_{\mathrm{p}} \gamma_{\mathrm{p}}$, the shape of the $\beta_{\text {app }}$ distribution function is nearly independent of the Lorentz factor.

3.2. Separate Pattern Velocities and Bends. If there were a ratio $r=\gamma_{\mathrm{p}} / \gamma_{\mathrm{b}} \neq 1$ between the Lorentz factors of the bulk flow and the radio knots (traveling or standing shocks, for example), Doppler beaming selection would favor angles inside $(r<1)$ or outside $(r>1)$ those at which the largest $\beta_{\text {app }}$ occur. Thus, for both slow and fast patterns (compared to the bulk flow), there usually would be a less pronounced upper $\beta_{\text {app }}$ envelope and a larger fraction of relatively slower motions. Fortunately, with careful Monte Carlo modeling (21) it will be possible to break the degeneracy between slow and fast pattern statistics by taking into account other measurements of the bulk Doppler factor, such as provided by the inverseCompton x-ray deficit (18).

The formalism for separate pattern velocities, with the same conclusions, also applies when there is acceleration or deceleration of the jets between the core area and the region where the moving knots are observed. However, at least in the case of 3C345, where apparent acceleration is actually observed, it is though to be due to bending of a constant- $\gamma$ jet (7-9). In that case, the model predictions are somewhat altered; the details are complicated (21). For small characteristic bends $\left(<1 / \gamma_{p}\right)$ in random azimuth, the fraction of the fastest $\beta_{\text {app }}$ actually increases, and the upper envelope becomes sharper. For larger bends, there is still a predominant velocity range, but it moves down from the maximum $\beta_{\mathrm{app}}=\beta_{\mathrm{p}} \gamma_{\mathrm{p}}$, so that there is then a large fraction of midsize motions, and the $\beta_{\text {app }}$ distribution has tails to both high and low values.

3.3. Potential for Cosmology. Observed $\beta_{\text {app }}$ values are inversely proportional to the value of $H_{0}$ assumed, while the predicted $\beta_{\text {app }}$ values in a sample selected on beamed emission are directly proportional to the Lorentz factor distribution over the sample. Therefore, if a value for $H_{0}$ is assumed, the $\gamma$-distribution can be measured, but if the $\gamma$-distribution can be estimated independently (for example using Doppler factors), then $H_{0}$ can be measured.
At higher redshifts, values of $\beta_{\text {app }}$ calculated from observed internal proper motions also depend on $q_{0}$; lower values of $q_{0}$ imply higher $\beta_{\mathrm{app}}$. One could assume that the superluminal motion phenomenon, as it is found in parsec-scale jets, does not evolve with cosmological epoch, since the large-scale environment does not play a direct role. The requirement that there be a standard apparent velocity distribution (a variant on the standard measuring rod) then would determine $q_{0}$. More generally, even if there were some evolution of the $\gamma$-distribution, incorrect low or high values for $q_{0}$ would progressively stretch or compress the $\beta_{\text {app }}$ distribution towards higher redshifts in a way that would not mimick simple forms of evolution of the Lorentz factor distribution. However, a meaningful constraint on $q_{0}$ will probably take several hundred apparent velocities, properly distributed to allow a determination of the Lorentz factor distribution in many narrow redshift bins.

\section{First Results from the CJ Survey}

The combination of the Pearson-Readhead (37) (PR) and California Institute of Technology-Jodrell Bank (CJ) VLBI (very-long-baseline radio interferometry) surveys (38-40) yields a complete flux-limited sample of 294 flat-spectrum sources brighter than $0.35 \mathrm{Jy}$ at $5 \mathrm{GHz}$. Second epoch observations, with a time interval of 2-3 years, are in progress, and hopefully will be completed in 1995. Apparent proper motions or useful upper limits are now available for 81 of these sources, after temporarily putting aside $\sim 1 / 3$ of the objects, in which the morphological changes, if any, are more ambiguous (mostly those with rather featureless or extended jets). The new motions were derived by fitting to the data at each epoch the relative positions and flux densities of a few Gaussian components with fixed shapes, determined in preliminary iterations. Where more than one motion could be measured in a source with multiple components, the value used is that between the two brightest features. The apparent velocities derived for both $q_{0}=0.5$ and $q_{0}=0.05$ are shown in Fig. 3 .

4.1. Galaxies, BL Lacs, and Quasars. Ignoring upper limits and empty fields, the mean apparent velocity is slightly smaller for the galaxies $\left(N=7,\left\langle\beta_{\text {app }}\right\rangle=2.1 \mathrm{~h}^{-1}\right)$ and the BL Lacs $(N$ $\left.=8,\left\langle\beta_{\text {app }}\right\rangle=2.3 \mathrm{~h}^{-1}\right)$ than for the quasars $\left(N=44,\left\langle\beta_{\text {app }}\right\rangle=\right.$ 


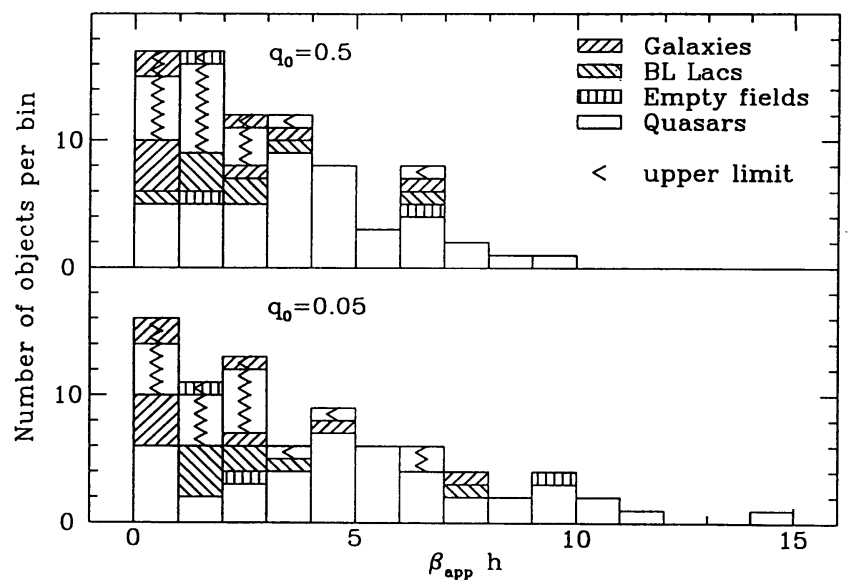

FIG. 3. The observed apparent velocity distribution for 81 objects in the homogeneous PR + CJ flat-spectrum sample, showing the scarcity of higher values, compared with earlier work and with predictions for beamed samples. There is no firm evidence for differences related to optical identification.

$3.2 \mathrm{~h}^{-1}$ ), all for $q_{0}=0.5$. This is in the sense expected for unification models. However, the Kolmogorov-Smirnov (KS) test yields a probability of $23 \%$ that the galaxies and the quasars have the same $\beta_{\text {app }}$ distribution and a probability of $30 \%$ for the BL Lac objects and the quasars. In short, the best current homogeneously selected data base provides no firm evidence for an assertion that there have to be different populations corresponding to galaxies, BL Lacs $(29,30)$, or quasars. With the full sample, stronger tests of unification models will be possible.

4.2. Scarcity of Fast Apparent Motions. The upper cutoff is not nearly as sharp as predicted in the simplest model discussed in section 3.1, and the apparent velocities do not cluster near the maximum; this is the same conclusion as that derived from an earlier more heterogeneous group of 25 core-selected quasars (21). There is a substantial fraction $(\sim 25 \%)$ of stationary features or upper limits. Then, if $q_{0}=0.5$ it seems that $\beta_{\text {app }}$ values in the range $1-5 h^{-1}$ occur with roughly equal frequency, with a tail $(17 \%)$ of higher values, up to $10 h^{-1}$. If $q_{0}=0.05$, the $\beta_{\text {app }}$ distribution tapers off even more gently; most values are still below $10 h^{-1}$.

This detailed distribution differs from that in the group analyzed earlier (21), of which, with $q_{0}=0.5,36 \%$ had $\beta_{\text {app }}=$ $5-10 h^{-1}$, more than twice the fraction in the new sample. The scarcity in $\mathrm{CJ}$ of these somewhat faster motions is certainly not an artefact caused by undersampling in time. It seems that the superluminal quasars reported thus far in the earlier literature are not representative of the population as a whole. There is not an obvious correlation of $\beta_{\text {app }}$ with flux density in the new sample, so other factors must be at work. Clearly, there was a bias in earlier work towards analyzing and publishing fast superluminals. Furthermore, a lot of the sources previously studied are highly variable [many appear in the Variable Source Sample (41), for example], and there is already evidence for a correlation between variability at high frequency and a large apparent velocity (12).

4.3. Plausible Lorentz Factor Distributions. Setting aside the upper limits and adopting $q_{0}=0.5$ (the least extreme case), the $\beta_{\text {app }}$ distribution can be reproduced by assuming that there is a wide range of Lorentz factors in the sample, with in particular a long tail to high values. As a numerical example for illustrative purposes only, if $h=0.55$ then $\gamma_{\mathrm{b}} \approx 4$ could be the peak of a skewed bell-shaped distribution spanning the range $\gamma_{\mathrm{b}} \approx 2-18$. Interestingly, such a distribution is akin to that derived from largely independent data, involving radio source counts (19).
Well-fitting models can also be found in which the observed patterns have a different Lorentz factor than the bulk flow. For $q_{0}=0.5$, one would need either $r=\gamma_{\mathrm{p}} / \gamma_{\mathrm{b}} \approx 0.25$ or $r \approx 10.0$; intermediate values are ruled out, unless there is also a considerable range of $\gamma_{\mathrm{b}}$ values. Such low or high $r$ are unappealing; $r$ $=0.25$ because, with $\beta_{\text {app }}$ up to $10 h^{-1}$, it requires a rather high $\gamma_{\mathrm{b}} \geq 40 h^{-1}$ in all objects; $r=10$ because, conversely, it requires that almost all objects have $\gamma_{\mathrm{b}} \leq 1 h^{-1}$, in contradiction with other evidence for substantial Doppler beaming. It seems that this high $r$ case is akin to a recent incarnation of the light-echo models (42), in which relativistic motion is admitted, but Doppler beaming is not. While it is entirely plausible that pattern velocities do play a role, for example in causing apparently stationary patterns in relativistic jets, further evidence that Doppler beaming is in fact important is given by the observed luminosity dependence of the $\beta_{\text {app }}$ distribution.

4.4. Luminosity Dependence. Fig. 4 shows the dependence of the observed $\beta_{\text {app }}$ on observed monochromatic luminosity $P_{5 \text {,obs, calculated by assuming isotropic emission. While low }}$ $\beta_{\text {app }}$ can be found at any observed luminosity, there seems to be a striking correlation of the largest $\beta_{\text {app }}$ with observed luminosity: the upper envelope rises considerably. However, it will be very desirable to verify this by measuring internal proper motions in a VLBI sample with a considerably wider spread of flux densities, so as to improve the coverage of lower observed luminosities and also to break the strong redshiftflux density correlation.

Fig. 4 suggests that most or all of our flat-spectrum sources at the high observed luminosity end $\left(P_{5, \mathrm{obs}} \sim 10^{34} \mathrm{erg} \cdot \mathrm{s}^{-1} \cdot \mathrm{Hz}^{-1}\right.$; $1 \mathrm{erg}=0.1 \mu \mathrm{J}$ ) conform roughly to the simple model: they have highly relativistic jets and are in our sample because their observed luminosity is considerably enhanced by Doppler beaming. Most of them are from a parent population 2 or 3 orders of magnitude down in intrinsic (isotropic) luminosity. Furthermore, the absence of similarly high $\beta_{\text {app }}$ at $P_{5, \text { obs }} \leq 10^{32}$ $\mathrm{erg} \cdot \mathrm{s}^{-1} \cdot \mathrm{Hz}^{-1}$ suggests that those objects are rather less beamed, which in turn implies that there is no substantial population down another 2 or 3 orders of magnitude in intrinsic luminosity, from which members can get Doppler beamed up. Thus, it would seem that highly relativistic jets may occur only in objects with a restricted range of $5-\mathrm{GHz}$ intrinsic luminosities, perhaps predominantly near $10^{31} \mathrm{erg} \cdot \mathrm{s}^{-1} \cdot \mathrm{Hz}^{-1}$, and that there is a correlation between intrinsic $5-\mathrm{GHz}$ radio luminosity and Lorentz factor, akin to that often postulated for the low-frequency radio luminosity (e.g. the FR-I-FR-II division). Clearly, further analysis of the effects seen in Fig. 4 is

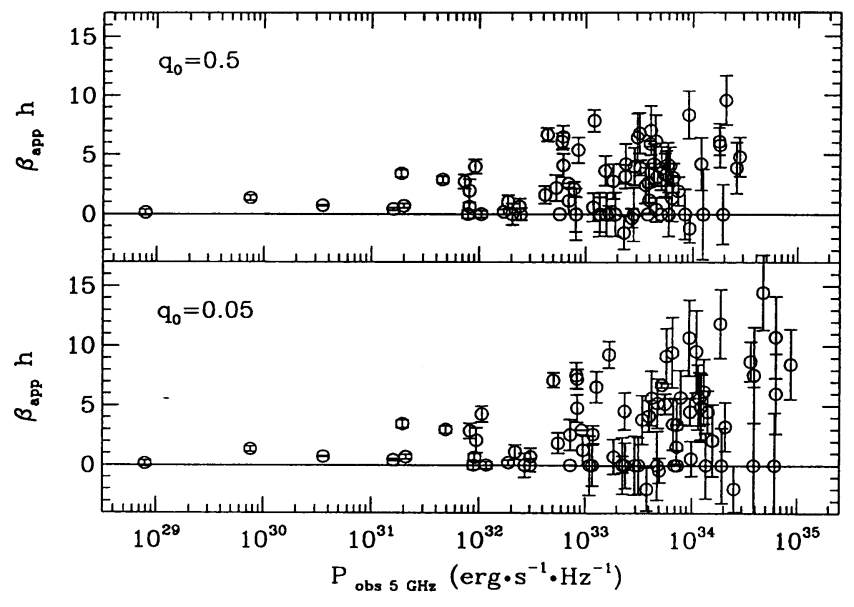

FIG. 4. The observed apparent velocity distribution for 81 objects in the homogeneous PR + CJ flat-spectrum sample, illustrating that the upper envelope rises as a function of the observed $5-\mathrm{GHz}$ monochromatic luminosity. Upper limits are plotted as error bars on $\beta_{\text {app }}=0$. 
needed, and a combination of source counts and the apparent velocity statistics of a larger sample could provide much insight into the properties of radio jet sources.

This review builds on earlier work done in collaboration with Marshall Cohen as cited. It includes many new measurements of the cited CJ VLBI survey sources, which will be published separately after further analysis. Wenge Xu, David Henstock, and Greg Taylor reduced most of the first-epoch data sets; Greg was also heavily involved in working on the second epochs and the motion measurements. The CJ survey has benefitted from generous allocations of time by the Global VLBI Network, involving the dedicated efforts of many people at the individual telescopes. The data were all correlated on the California Institute of Technology-Jet Propulsion Laboratory Block II correlator. The pace of the project would not have been so rapid without Martin Shepherd's DIFMAP (43). Tim Pearson is thanked for his stimulating role as the referee. This work has been supported in part by the National Science Foundation under Grants AST 88-14554, AST 91-17100, and AST 94-20018.

1. Kellermann, K. I., Clark, B. G., Bare, C. C., Rydbeck, O., Ellder, J., Kollberg, E., Hoglund, B., Cohen, M. H. \& Jauncey, D. L. (1968) Astrophys. J. Lett. 153, L209-214.

2. Moffet, A. T., Gubbay, J., Robertson, D. S. \& Legg, A. J. (1972) Int. Astron. Union Symp. 44, 228-229.

3. Whitney, A. R., Shapiro, I. I., Rogers, A. A. E., Robertson, D. S., Knight, C. A., Clark, T. A., Goldstein, R. M., Marindino, G. E. \& Vandenberg, N. R. (1971) Science 173, 225-230.

4. Cohen, M. H., Cannon, W., Purcell, G. H., Shaffer, D. B., Broderick, J. J., Kellermann, K. I. \& Jauncey, D. L. (1971) Astrophys. J. 170, 207-217.

5. Cohen, M. H., Kellermann, K. I., Shaffer, D. B., Linfield, R. P., Moffet, A. T., Romney, J. D., Seielstad, G. A., Pauliny-Toth, I. I. K., Preuss, E., Witzel, A., Schilizzi, R. T. \& Geldzahler, B. J. (1977) Nature (London) 268, 405-409.

6. Kellermann, K. I. \& Cohen, M. H. (1988) J. R. Astron. Soc. Can. 82, 248-265.

7. Zensus, J. A., Cohen, M. H. \& Unwin, S. C. (1995) Astrophys. J. 443, 35-53.

8. Wardle, J. F. C., Cawthorne, T. V., Roberts, D. H. \& Brown, L. F. (1994) Astrophys. J. 437, 122-135.

9. Zensus, J. A. (1995) Proc. Natl. Acad. Sci. USA 92, 11348-11355.

10. Alberdi, A., Marcaide, J. M., Marscher, A. P., Zhang, Y. F., Elosegui, P., Gomez, J. L. \& Shaffer, D. B. (1993) Astrophys. J. 402, 160-172.

11. Mutel, R. L., Phillips, R. B., Su, B. \& Bucciferro, R. R. (1990) Astrophys. J. 352, 81-95.

12. Teräsranta, H. \& Valtaoja, E. (1994) Astron. Astrophys. 283, 51-58.

13. Hughes, P. F., Aller, M. F. \& Aller, H. D. (1994) Astrophys. J. 396, 469-486.

14. Readhead, A. C. S. (1994) Astrophys. J. 426, 51-59.

15. Orr, M. J. L. \& Browne, I. W. A. (1982) Mon. Not. R. Astron. Soc. 200, $1067-1080$

16. von Montigny, C., Bertsch, D. L., Chiang, J., Dingus, B. L., Esposito, J. A., Fichtel, C. E., Fierro, J. M., Hartman, R. C., Hunter, S. D., Kanbach, G., Kniffen, D. A., Lin, Y. C., Mattox,
J. R., Mayer-Hasselwander, H. A., Michelson, P. F., Nolan, P. L., Radecke, H. D., Schneid, E., Sreekumar, P., Thompson, D. J. \& Willis, T. (1995) Astrophys. J. 440, 525-553.

17. Barthel, P. D., Conway, J. E., Myers, S. T., Pearson, T. J. \& Readhead, A. C. S. (1995) Astrophys. J. Lett. 444, L21-L24.

18. Ghisellini, G., Padovani, P., Celotti, A. \& Maraschi, L. (1993) Astrophys. J. 407, 65-82.

19. Padovani, P. \& Urry, C. M. (1992) Astrophys. J. 387, 449-457.

20. Cohen, M. H., Barthel, P. D., Pearson, T. J. \& Zensus, J. A. (1988) Astrophys. J. 329, 1-7.

21. Vermeulen, R. C. \& Cohen, M. H. (1994) Astrophys. J. 430, 467-494.

22. Pelletier, G. \& Roland, J. (1989) Astron. Astrophys. 224, 24-30.

23. Readhead, A. C. S. (1995) Proc. Natl. Acad. Sci. USA 92, 1138111384.

24. Taylor, G. B., Vermeulen, R. C. \& Pearson, T. J. (1995) Proc. Natl. Acad. Sci. USA 92, 11381-11384.

25. Hough, D. H. (1994) in Compact Extragalactic Radio Sources, eds. Zensus, J. A. \& Kellermann, K. I. (National Radio Astronomy Observatory, Charlottesville, NC) p. 169-174.

26. Zensus, J. A. \& Porcas, R. W. (1987), in Superluminal Radio Sources, eds. Zensus, J. A. \& Pearson, T. J. (Cambridge Univ. Press, Cambridge, U.K.) p. 126-128.

27. Scheuer, P. A. G. \& Readhead, A. C. S. (1979) Nature (London) 277, 182-185.

28. Vermeulen, R. C., Bernstein, R. A., Hough, D. H. \& Readhead, A. C. S. (1993) Astrophys. J. 417, 541-546.

29. Gabuzda, D. C., Mullan, C. M. \& Cawthorne, T. V. (1994) Astrophys. J. 435, 140-161.

30. Gabuzda, D. C. (1995) Proc. Natl. Acad. Sci. USA 92, 1139311398.

31. Laing, R. A. (1993), in Astrophysical Jets, eds. Burgarella, D., Livio, M. \& O’Dea, C. P. (Cambridge Univ. Press, Cambridge, U.K.), p. 95-114.

32. Gopal-Krishna \& Wiita, P. J. (1993) Nature (London) 363, 142144.

33. Urry, C. M., Padovani, P. \& Stickel, M. (1991) Astrophys. J. 382, 501-507.

34. Laing, R. A. (1995) Proc. Natl. Acad. Sci. USA 92, 11413-11416.

35. Barthel, P. D. (1989) Astrophys. J. 336, 606-611.

36. Lind, K. R. \& Blandford, R. D. (1985) Astrophys. J. 295, 358-367.

37. Pearson, T. J. \& Readhead, A. C. S. (1988) Astrophys. J. 328, 114-142.

38. Polatidis, A. G., Wilkinson, P. N., Xu, W., Readhead, A. C. S., Pearson, T. J., Taylor, G. B. \& Vermeulen, R. C. (1995) Astrophys. J. Suppl. 98, 1-32.

39. Taylor, G. B., Vermeulen, R. C., Pearson, T. J., Readhead, A. C. S., Henstock, D. R., Browne, I. W. A. \& Wilkinson, P. N. (1994) Astrophys. J. Suppl. 95, 345-369.

40. Wilkinson, P. N. (1995) Proc. Natl. Acad. Sci. USA 92, 1134211347.

41. Wehrle, A. E., Cohen, M. H., Unwin, S. C., Aller, H. D., Aller, M. F. \& Nicolson, G. (1992) Astrophys. J. 391, 589-607.

42. Ekers, R. D. \& Liang, H. (1990) in Parsec-Scale Radio Jets, eds. Zensus, J. A. \& Pearson, T. J. (Cambridge Univ. Press, Cambridge, U.K.) p. 333-339.

43. Shepherd, M. C., Pearson, T. J. \& Taylor, G. B. (1994) Bull. Am. Astron. Soc. 26, 987. 